\title{
EL FRENTE NACIONAL: LEGITIMIDAD INSTITUCIONAL Y CONTINUISMO BIPARTIDISTA EN COLOMBIA (1958-1974)'
}

\author{
Álvaro Acevedo Tarazona ${ }^{2}$ \\ Universidad Industrial de Santander, Colombia - Artículo Tipo 1. \\ Investigación Científica y Tecnológica - Recibido: 10 de Abril 2015 - Aceptado: 13 de Junio 2015 \\ doi: http://dx.doi.org/10.17981/econcuc.36.1.2015.22
}

\section{RESUMEN}

Mediante un análisis histórico de perspectiva contextual, enfoque que permite la reconstrucción de las principales características sociales, políticas, culturales y económicas de una época; este artículo centra su atención en los elementos que posibilitaron el surgimiento del Frente Nacional como pacto bipartidista, además de considerar las acciones que propició el surgimiento de nuevos obstáculos al desarrollo de la democracia en Colombia. Mientras que la violencia de los años cincuenta y la dictadura del General Gustavo Rojas Pinilla, fueron identificadas como causas del surgimiento de la nueva estructura política del país, la exclusión política de actores sociales inéditos como el movimiento campesino, o recientes como el movimiento obrero y el movimiento estudiantil, fue percibida como su principal consecuencia. Como resultado del análisis, este estudio permitió configurar una nueva hipótesis en torno al significado histórico del Frente Nacional, al concluir que, si bien es cierto que el frente nacionalista ayudó a fortalecer las instituciones políticas y económicas del país, al legalizar la discriminación de cualquier otra fuerza social que pretendiera representar los intereses ciudadanos, el pacto propiciaba la aparición de un nuevo problema histórico para la democracia colombiana: la exclusión de los actores políticos de oposición, un aspecto aún vigente en esta democracia.

Palabras Clave:

Frente Nacional, Dictadura, Plebiscito. JEL: D74, D78, F52

\section{Si va a referenciar este artículo}

Acevedo, A. (2015). El Frente Nacional: Legitimidad institucional y continuismo bipartidista en Colombia (1958-1974), Económicas CUC, 36(1), 49-78.

doi: http://dx.doi.org/10.17981/econcuc.36.1.2015.22

\footnotetext{
${ }^{1}$ El presente artículo es producto de la investigación: "La experiencia histórica del "cogobierno" en la Universidad Industrial de Santander: concepciones y divergencias en disputa por la autonomía universitaria, 1971-1976”, financiado por la Vicerrectoría de Investigación de la Universidad Industrial de Santander
}

${ }^{2}$ Doctor en Historia de la Universidad de Huelva. Docente Titular de la Universidad Industrial de Santander, Bucaramanga., Director del Grupo de Investigación "Políticas, Sociabilidades y Representaciones Histórico-Educativas (PSORHE)", acetara@uis.edu.co 


\section{INTRODUCCIÓN}

En el presente artículo se describe y analiza la historia del Frente Nacional (FN), el acuerdo político bajo el cual vivió el país entre 1958 y 1974. El análisis se ocupa tanto de su origen, como de su desenvolvimiento y legado. No solo se examina su origen legal, a través del plebiscito de 1957, sino las circunstancias sociales y políticas que configuraron el contexto en el que apareció como una respuesta inevitable a la violencia política de mediados del siglo XX, así como a la dictadura del General Gustavo Rojas Pinilla. Si bien se reconoce que el FN fue una estrategia que permitió fortalecer las instituciones políticas y económicas del país, no hay que perder de vista que su modus operandi-caracterizado por el reconocimiento legal de las dos fuerzas políticas tradicionales: los partidos liberal y conservador-creó nuevos y acuciantes problemas nacionales, no sólo al legalizar la discriminación de los actores políticos que no se identificaban con los partidos tradicionales, sino al combatir cualquier fuerza que pretendiera ganarse el reconocimiento político de la sociedad.

En efecto, tal como se describe a lo largo del texto, el FN permitió contener la violencia y reencausar al país por la senda de la democracia, pero dejó por fuera de su alcance una serie de aspectos que también merecían su atención, como la apertura de nuevos caminos de participación política para los sectores sociales que el desarrollo económico "sacaba a flote". El texto intenta argumentar, en consecuencia, que si bien el FN marcó la vida política del país, no sólo lo hizo porque redujera ostensiblemente la violencia política que "desangraba" al país, sino porque logró excluir y vencer a las fuerzas políticas que quisieron erigirse en los nuevos representantes de la nación y todo ello a través de su principal fórmula: la alternancia y la paridad entre los partidos tradicionales. De hecho, el movimiento estudiantil, la lucha obrera y la organización de nuevos grupos políticos, influenciados sin duda por lo que acontecía en el mundo, pero también impulsados por sus propias fuerzas; intentaron infructuosamente, ganar visibilidad política y social durante el $\mathrm{FN}$, uniendo e incluso a un líder populista como Rojas Pinilla, el mismo a quien el bipartidismo oficial había desplazado para recuperar la democracia.

\section{DESARROLLO}

\section{Las condiciones de posibilidad del Frente Nacional}

Colombia no ha sido el único país en apelar a los pactos bipartidistas para dar solución a sus problemas de institucionalidad y debilidad estatal. Uruguay y Venezuela en las décadas de 1950 y 1960, y Chile y Argentina en los años ochenta, solo para señalar algunos casos de América Latina, vivieron algo semejante durante los periodos de transición o de reconquista de sus democracias. Para analizar este tipo de hechos y para comprender el caso colombiano Hartlyn (1993), utilizó el concepto politológico de consociacionalismo ${ }^{3}$, entendiendo por tal al fenómeno político por el cual las clases dirigentes tradicionales conforman coaliciones que facilitan la transición hacia un gobierno democrático, pero restringiendo de cierto modo la misma democracia.

\footnotetext{
3 Consociacionalismo se refiere por lo general a una forma de gobierno recomendada para el manejo de conflictos en sociedades profundamente divididas. A menudo se considera sinónimo de compartir el poder.
} 
Con todo, no debe creerse que dicho fenómeno sea el mismo en todos los países. Por el contrario, debe reconocerse que en cada país, dadas sus peculiaridades políticas, el consociacionalismo se desarrolló de manera distinta.

Por consiguiente, la primera pregunta que debemos intentar resolver para poder entender esta etapa de nuestra historia reciente es ¿cuáles fueron los hechos que llevaron a que tal pacto frentenacionalista fuera planteado como una medida de transición hacia la democracia en Colombia?

Sin duda, un juego de complejas circunstancias históricas hizo del pacto del Frente Nacional una necesidad. En efecto, la violencia y la dictadura del General Rojas Pinilla habían hundido al país en un profundo "pozo" de ilegitimidad. Pactar un nuevo orden político se hizo en consecuencia inevitable. Fue por esta razón que se propuso poner a consideración de la sociedad un proyecto político conciliador, pues sólo a través de un plebiscito, tal como lo ha expresado Calle (2012), podría la nación "recuperar el hilo constitucional" que tras la violencia y la dictadura había perdido.

La Violencia con V mayúscula, es decir, concebida como una cruenta etapa de la historia colombiana en que liberales y conservadores se "despachaban" el país sin más razón que el odio mismo; como se sabe, fue desencadenada por el asesinato de Jorge Eliecer Gaitán, ocurrido el 9 de abril de 1948. Ciertamente, fue ese el acontecimiento que hizo visible la profunda brecha que separaba en dos bandos a las élites políticas y al pueblo llano. "El asesinato de Gaitán -nos cuenta Henderson (2006, p. 452)- lanzó a sus seguidores a un frenesí" de venganza. El odio, como el fuego, había sido avivado.
Estos hechos eran el preludio de una guerra civil que después de 1949 solo conocería breves e inexplicables lapsos de paz bipartidista, pues el panorama general estaría dominado por la intransigencia y la mutua enemistad, hasta desembocar nada menos que en la suspensión del gobierno democrático. Hacia junio de 1949 los liberales se presentaron a las elecciones legislativas con la plena seguridad de que serían mayoría en el congreso, y de que lograrían hacerle una fuerte oposición al gobierno conservador del Dr. Mariano Ospina Pérez, a quien culpaban de la Violencia, porque lo consideraban débil y carente del liderazgo necesario para evitar que el pueblo se desangrara. No obstante, y pese a que constituyeron mayoría en el legislativo, la estrategia no resultó, pues la constitución política facultaba al poder ejecutivo para disolver el Congreso en caso de que el orden público se hallara perturbado. Fue esto precisamente lo que Ospina hizo el 9 de noviembre de 1949: declarar el estado de sitio y suspender el Congreso. Ante tamaña acción, los liberales consideraron que solo les quedaba un camino: armar sus guerrillas para conseguir por la fuerza lo que no habían logrado por la vía política.

A partir de ese momento, recurriendo aquí al concepto propuesto recientemente por Palacios (2012, p. 25), no cabe duda que Colombia se enfrascaba en la primera -y en una de las peores-oleadas de "violencia pública", es decir, de ese tipo de violencia tanto social como estatal que requiere de "un discurso de autolegitimación" que justifique la "tragedia de miles de hogares y vecindarios", el quebrantamiento de "los códigos morales" y el cercenamiento de los lazos sociales del país. 
En todo caso, aunque el comportamiento del fenómeno de la violencia fue disímil, no cabe duda que mantenía en estado de máxima alerta a todo el país. En distintas regiones, pero principalmente en los Llanos orientales y la zona cafetera, territorios en los que el estado a duras penas podía llegar con su brazo armado, las bandas y las guerrillas de origen liberal, en un principio, y comunista, tiempo después, empezaron a hacer presencia, a controlar la situación y a hacer de las suyas eliminando a sus contrincantes y constriñendo la voluntad popular durante los periodos electorales (Henderson, 1984). Pero además de estos hechos, a todas luces verídicos, el país empezó a experimentar también el mito de la Violencia. En efecto, dice Palacios (1998), que los episodios de violencia ocurridos entre 1945 y 1964 fueron convertidos por la tradición oral en una "colección de testimonios más o menos verídicos, transformados en leyendas fragmentarias por la incontable sucesión de narradores. Los años 50 y 60 dieron aliento a una ola de ensayos, novelas, representaciones teatrales, producciones cinematográficas y creaciones de las artes visuales" que difundieron la "leyenda negra' de la Violencia en Colombia.

Ahora bien, a estos hechos se sumaba un panorama político cada vez más oscuro. En 1950, tras declarar el estado de sitio y llevarse a cabo las elecciones, el Dr. Ospina Pérez entregó el poder al conservador Dr. Laureano Gómez. Este se había propuesto conjurar la violencia a través de una reforma constitucional que reconsideraba, entre otros aspectos, las funciones del poder legislativo, le devolvía a la iglesia un papel preponderante en el control de la educación, limitaba la libertad de expresión y le quitaba al congreso la facultad de legislar sobre las funciones de las fuerzas armadas. Aunque la reforma jamás dejo de ser un proyecto, fue mal recibida por el liberalismo, e incluso un sector del conservatismo desconfió también de ella. Así entonces, desde muy temprano el gobierno de Gómez se granjeaba el aborrecimiento tanto de liberales como de sus propios partidarios. Laureano Gómez, nos dice Palacios (1998, p.210-211), "no comprendió que los límites de su autoritarismo estaban trazados de antemano", y que su "gobierno excluía demasiados intereses", principalmente los de las Fuerzas Armadas, así que no vio venir el cuartelazo que acabó con su gobierno. Y en efecto, "en la noche del 13 de junio de 1953, y con el respaldo del mismo Dr. Ospina y la plana mayor de la oposición conservadora, el General Rojas Pinilla anunció la consumación de un golpe de estado. La iglesia, los gremios empresariales y todos los grupos políticos, con la excepción de un puñado de laureanistas y del partido comunista, lo avalaron". (Palacios, 1998, p. 211)

El golpe militar fue recibido con beneplácito por varios sectores políticos; algunos incluso recibieron a Rojas como el salvador de la nación (Tirado, 1981). Para dar mayores visos de legitimad, Rojas y sus seguidores organizaron una Asamblea Nacional Constituyente con el fin de establecer, por un lado, que el 13 de junio había quedado vacante la presidencia, y por otro, que Rojas tomaría posesión del cargo mientras se reorganizaban las instituciones. Su gobierno se caracterizó por los siguientes aspectos: tan pronto como ascendió al poder y con la finalidad de asegurar su reelección el general empezó una maratónica gira proselitista por todo el país; conformó una nómina de gobierno que incluía, aunque no de manera equitativa, a liberales y conservadores, 
siendo estos últimos los más numerosos; solucionó el conflicto llanero, reduciendo el número de muertes por la violencia de 22.000 en $1952-1953$ a 1.900 en 1954 1955, y atacó la corrupción judicial mediante el despido de funcionarios afectos al laureanismo. (Henderson, 2006)

En abril de 1954, Gustavo Rojas Pinilla convocó nuevamente la Asamblea Nacional Constituyente, la cual había sido disuelta en junio de 1953, después de legalizar el acenso de Rojas al poder. El objetivo de la nueva convocatoria era anunciar que el país aún no contaba con las condiciones de orden público adecuadas para realizar las elecciones presidenciales, así como manifestar que se comprometía a dejar el poder tan pronto el orden retornara. Entre tanto, Rojas instituyó el sufragio femenino; proscribió constitucionalmente al partido comunista y reemplazó las Asambleas Departamentales y los Consejos Municipales por sendos cuerpos administrativos. En agosto de 1954 la Asamblea Nacional Constituyente prorrogó el mandato de Rojas hasta 1958. Para los liberales esta había sido la jugada política más atrevida de Rojas y desde sus plataformas de combate, los diarios El Tiempo y El Espectador, decidieron contenerla. El FN respondió ordenando la clausura de los periódicos. Fue entonces cuando la dictadura militar se hizo evidente y pese a que bajo la "égida" del General Rojas el país estaba viviendo mejores tiempos -la bonanza cafetera y la estabilidad monetaria daban cuenta de ello, como lo ha mostrado Kalmanovitz (1995)- no se requerían complicadas razones para argumentar que la prorroga significaba un mayor debilitamiento de la democracia.

Los partidos tradicionales no dudaron entonces en retirar al líder militar el apoyo que le prodigaran en 1953. Tam- poco dudaron en hacer eco de las demandas que una buena parte de la sociedad venía realizando por lo menos desde 1954, con respecto a las libertades de expresión y de movilización. No obstante, la gota que derramó la copa fue la ostensible manifestación de fuerza que el general Rojas Pinilla llevó a cabo el 13 de junio de 1956, en el estadio de fútbol de la capital, cuando presentó ante la multitud el partido político que había fundado y al que denominó la Tercera Fuerza. $\mathrm{El}$ evento fue todo un ritual, pues "miles de asistentes" desfilaron "ante el "Jefe Supremo»", quien se hallaba ataviado con "todas sus galas y condecoraciones", mientras juraban por Dios que le serían leales al pueblo y a las fuerzas armadas (Palacios, 1998, p.215-216). Si a un hecho como este se le sumaban -como perlas- el asesinato de un estudiante de la Universidad Nacional ocurrido el 8 de junio de 1954 a manos del batallón Colombia, o la imposición de un gravamen a los beneficios de los industriales, hechos que tanto repudiaron los estudiantes y los gremios, no había duda que la clase dominante necesitaba una nueva salida. Así pues, como nos dice Tirado (1981), los dirigentes de los dos partidos tradicionales, no tuvieron otra opción que ponerse de acuerdo y unir sus fuerzas para combatir al enemigo común. La dictadura militar de Rojas fue, por consiguiente, el segundo de los elementos contextuales que posibilitarían el surgimiento del Frente Nacional.

\section{El desenvolvimiento del Frente Nacional}

El plebiscito que dio vida al Frente $\mathrm{Na}$ cional se llevó a cabo el lunes primero de diciembre de 1957. Aquel día, por primera vez en la historia republicana de Colombia, las mujeres asistían en masa 
a las urnas. Hacia el final de la jornada el ex presidente Alberto Lleras Camargo confirmaba que el documento del acuerdo entre los partidos había sido refrendado por el $94,8 \%$ de los votantes, es decir, por 4.169 .294 votos. Solo el $4,7 \%$ de los ciudadanos votó en contra. Si se tiene en cuenta, además, que en el evento participó el $81,95 \%$ del censo electoral, no cabe duda que el Frente Nacional, en efecto, obtuvo la legitimidad democrática que los partidos esperaban. (Calle, 2012)

¿Qué votaron favorablemente los colombianos? El documento del acuerdo señalaba lo siguiente (Plazas, 2011):

1. Le otorgaba a las mujeres los mismos derechos políticos que ya tenían los varones.

2. Establecía la paridad de cargos, tanto de elección popular como de nombramiento administrativo, entre los partidos conservador y liberal.

3. Señalaba que, para dirimir impases en cualquier corporación, se consideraba que la mayoría estaría conformada por las dos terceras partes.

4. Le reconocía al Presidente de la República el derecho a nombrar y remover libremente a los miembros de su gabinete, pero le obligaba a dar participación a los dos partidos políticos en la misma proporción en que estuvieran representados en las cámaras legislativas.

5. Otorgaba al Congreso de la República la facultad de establecer y regular las funciones y garantías de los empleados públicos.

6. Prohibía que los empleados y funcionarios públicos tomarán parte en actividades proselitistas, sin perjuicio de ejercer libremente el derecho al sufragio.
7. Señalaba que en ningún caso la filiación política de los ciudadanos podría determinar su nombramiento para un empleo o cargo público, ni para su destitución o promoción.

8. Indicaba que los miembros del Congreso y de las Asambleas Departamentales no tendrían sueldo permanente, sino asignaciones diarias durante el término de las sesiones; y

9. Establecía que el gobierno invertiría el $10 \%$ de su presupuesto general de gastos en la educación pública. Ordenaba que las reformas constitucionales solo las podría hacer el Congreso.

Tras resultar vencedor en las elecciones del 4 de mayo de 1958, el Dr. Alberto Lleras Camargo recibía un país que aún era azotado por la violencia. Si bien las tasas de homicidios no llegaban a los picos presentados durante su primera fase (1947-1953), el conflicto todavía sacrificaba a miles de colombianos (Henderson 2006, Anexo 2). Así pues, para acelerar el proceso de pacificación del país, el nuevo presidente decidió, por un lado, mantener el programa de amnistía e indultos que la Junta Militar había instaurado; y por otro, poner en marcha un comité de rehabilitación de las víctimas de la violencia. Sus esfuerzos tuvieron éxito relativo. Un año después, la reducción de las muertes relacionadas con la violencia política fue significativa-un tercio menos en relación con el año anterior- pero esto indicaba que aún morían por esta causa poco menos de 2000 personas al año.

En efecto, el país empezaba a experimentar nuevos y graves problemas socio-económicos, como el crecimiento demográfico, la crisis cambiaria, el déficit fiscal y la desigualdad social debido a la concentración de la propiedad de la tie- 
rra. Con respecto al crecimiento demográfico, puede señalarse lo siguiente. Se trataba de un fenómeno global que afectaba tanto al campo como a la ciudad, y que alarmaba no solo a las élites locales sino a los técnicos extranjeros que asesoraban a los nuevos gobiernos. El mismo Dr. Lleras Camargo consideraba que el país no estaba preparado para diseñar políticas dirigidas a contener el rápido crecimiento demográfico. En consecuencia, como la iglesia y el partido conservador ejercían una fuerte presión para evitar que el estado implementara y dirigiera programas modernos para el control de la natalidad, los gobiernos frente nacionalistas debieron dejar en manos de entidades privadas la aplicación de tecnologías modernas de control natal. La medida, se quiera o no reconocer, resultó buena, pues, en última instancia, evitó que el programa se politizara, con lo cual llegó a la gran mayoría de la población. (Urrutia, 2012)

La crisis cambiaria, por su parte, iniciada en 1957, apenas pudo ser contenida hacia 1960, pues el fenómeno adquirió rápidamente un talante estructural y de mayor duración. Como la política de industrialización necesitaba de la importación de insumos industriales y agrícolas, y debido a que las exportaciones de materias primas crecían a un menor ritmo, la balanza de pagos siempre fue negativa. El fenómeno, común en casi toda América Latina, llevó a países como Colombia a adoptar el modelo de las dos brechas, un concepto económico de la época según el cual los países pobres debían, por un lado, aumentar sus exportaciones, y por otro, captar más ayuda internacional, puesto que se creía que los principales problemas de los países en desarrollo derivaban de la falta de divisas y ahorros. Como habrá de suponerse, el modelo de las dos brechas marcó la política econó- mica de todo el periodo, obligando a los gobiernos del Frente Nacional no sólo a concentrar sus esfuerzos administrativos en la gestión de los créditos externos, sino a organizar sus propios presupuestos en consonancia con los intereses de las agencias bancarias que accedían a inyectar las divisas que el país necesitaba. (Kalmanovitz, 1995)

Pero no todo era negativo, el pacto bipartidista, en medio de un ambiente en el que campeaban en América Latina las amenazas del totalitarismo de izquierda, le permitió a Colombia granjearse el favor de los EE.UU. Ciertamente, en el periodo del Frente Nacional la ayuda extranjera fluyó constantemente hacia Colombia, sobre todo a partir del gobierno del presidente Kennedy, quien, con "la intención de repetir el éxito del Plan Marshall 4 en Europa", creó para América Latina la Alianza para el Progreso. La ayuda externa se convirtió, por consiguiente, en un elemento central de la política económica y social de los gobiernos del Frente Nacional, en principio, porque era la única manera de acceder a créditos blandos y de largo plazo, y en última instancia, porque no se concebían más maneras de impulsar el desarrollo y fortalecer la estructura económica del país, puesto que, sin el aval de las agencias internacionales -como el Fondo Monetario Internacional y el Banco Mundial- la economía colombiana no habría alcanzado cierta estabilidad. (Urrutia, 2012)

\footnotetext{
4 Llamado oficialmente European Recovery Program o $E R P$ ) fue el plan más importante de Estados Unidos para la reconstrucción de los países europeos después de la Segunda Guerra Mundial, que a la vez estaba destinado a contener un posible avance del comunismo. La iniciativa recibió el nombre del Secretario de Estado de los Estados Unidos, George Marshall, y fue diseñada principalmente por el Departamento de Estado, en especial por William L. Clayton y George F. Kennan
} 
Con todo, el país jamás dejo de experimentar los altibajos propios de la economía de mercado. De hecho, aquella política macroeconómica fue objeto de varios y periódicos desequilibrios cambiarios, debido principalmente a que Colombia mantenía tasas de inflación superiores a las internacionales. Este hecho obligaba a los gobiernos a "realizar devaluaciones nominales periódicas", lo cual acarreaba costos políticos altos, pues se hacía necesario ajustar los precios de los productos que jugaban un papel central dentro de la economía, como los alimentos importados, la gasolina y el transporte. De ahí que, en gran medida -como lo han demostrado los estudios de Archila (2003), sobre la protesta en Colombia- no fueron pocas las manifestaciones protagonizadas por la sociedad civil, con el objeto de exigir el desmonte de aquellas regulaciones gubernamentales estrictamente económicas que terminaban perjudicando a los ciudadanos de a pie.

El tercer problema que debieron afrontar los gobiernos del Frente Nacional fue el constante déficit fiscal. Efectivamente, durante todo el periodo el país experimentó una profunda restricción fiscal. Hacia 1969, el Informe Musgrave señalaba que las arcas del país apenas acumulaban un $8 \%$ del producto interno bruto, pese a que los gobiernos del Dr. Lleras Camargo y el Dr. Guillermo León Valencia habían adelantado y fortalecido una reforma tributaria (Musgrave, 1969). La dificultad social que esto acarreaba se relacionaba con la imposibilidad del estado para hacerse cargo de la provisión de bienes y servicios públicos, de la misma manera que de los subsidios requeridos por el sector productivo (Junguito \& Rincón, 2007). La falta de recursos tributarios aumentaba la dependencia del crédito externo, y como este solo apoyaba las inversiones en infraestructura, el sector de los servicios quedaba prácticamente desahuciado. Solo el sector educativo, sin duda gracias a que el mismo plebiscito lo ordenaba, contaría con una bolsa de inversión mayor. Así pues, durante el Frente Nacional, la inversión pública en educación aumentó del 1\% del PIB entre 1938 y 1958 , al 3,5\% a principios de la década del 60. (Ramírez \& Tellez, 2007)

Los gobiernos del Frente Nacional también debieron lidiar con los graves problemas de desigualdad social debido a la concentración de la tierra. De ahí entonces que, desde la apertura del frente, nuevamente en la historia colombiana las élites políticas, empresariales y agrarias aceptaran hablar seriamente de la reforma agraria -ya en la república liberal se había tratado el tema-. Hacia los años 60, para la mayoría de los políticos -entre los que sobresaldrían los liberales- era una verdad incontestable que para acelerar el desarrollo económico el país requería de una reforma agraria. Ya en 1951 el Banco Mundial "había sostenido que la tierra de los latifundios estaba mal utilizada" y que "una reforma agraria no sólo mejoraría la distribución del ingreso, sino que también aceleraría el crecimiento de la producción agrícola" (Urrutia, 2012, p.249). Colombia, como muchos otros países del mundo, se caracterizaba -y se caracteriza aún- por una alta concentración de la tierra, con lo cual padecía de altísimos niveles de desigualdad. Así pues, aunque se trata de una opción pocas veces empleada -por obvias razones, ya que era los gobernantes quienes concentraban la tierra-, la reforma agraria fue consideraba una herramienta adecuada para subsanar tales desigualdades. No obstante, como bien lo ha demostrado Berry (2012, p.296) "los 
objetivos planteados durante [el Frente Nacional] no pudieron cumplirse".

En resumen, dicen los expertos, el Frente Nacional tuvo en la reforma agraria la solución "al conjunto de problemas que sufría el país durante el siglo XX”, no obstante, su inadecuada y débil aplicación constituyó su mayor fracaso (Berry, 2012, p.296). La iniciativa pretendía tanto redistribuir la tierra como propiciar la industrialización del sector agrario. Para impulsar esta iniciativa, el Dr. Lleras Camargo firmó la Ley 135 de 1961, con la cual se creaba el Instituto Colombiano de la Reforma Agraria (INCORA), un organismo que tendría a su cargo el proceso de titulación de tierras baldías y la búsqueda de mecanismos adecuados para hacer más productivo al agro colombiano. Puntualmente, la ley obligaba a los terratenientes, so pena de extinción de dominio, a modernizar los medios de explotación del suelo, así como a efectuar sobre él un uso más adecuado (Machado, 1998). Con todo, ni lo uno ni lo otro dio resultados duraderos, pues la implementación de la ley siempre fue obstaculizada por los grandes hacendados. Durante el gobierno del Dr. Valencia, por ejemplo, si bien la reforma agraria no fue enteramente suspendida, experimentó una profunda desaceleración. Con la victoria de Carlos Lleras Restrepo, quien había impulsado la ley desde el Congreso durante el gobierno del Dr. Lleras Camargo, la medida nuevamente cobró vitalidad. En esta ocasión, el estado se preocupó por organizar al campesinado colombiano a través del Decreto 755 de 1967, que ordenaba realizar un registro de todos los campesinos usuarios de servicios estatales, de manera que se pudiera contar con información fidedigna sobre el tamaño de este sector social.
Sin embargo, con el retorno de los conservadores al poder en 1970, la norma, y con ella la anhelada ilusión de los campesinos colombianos de alcanzar una mayor igualdad e inclusión social, encontraba su fin. En efecto, pese que para esta época el proceso de modernización agrícola había empezado a andar, antes de terminar el periodo del Dr. Misael Pastrana, el gobierno y los grandes propietarios acordaron abandonar definitivamente la reforma (Berry, 2012). En conclusión, a lo largo de todo el periodo del Frente Nacional el "proceso reformista se vio obstaculizado por factores políticos, económicos y jurídicos, y otros de carácter operativo y técnico" que lo hicieron inviable. (Machado, 2011)

Cabría describir, los principales hechos que a lo largo del periodo protagonizaron los nuevos actores sociales: el estudiantado y las agrupaciones políticas que el FN dejaba por fuera del juego político al desconocer la oposición Ayala (1999). Luego de la caída de Gustavo Rojas Pinilla y de la transición hacia el "régimen" bipartidista, la sociedad colombiana vio emerger con una fuerza considerable a los jóvenes universitarios. Como se ha señalado, el FN y su estructura burocrática paritaria si bien permitió la disminución y erradicación de la violencia bipartidista y luego bandolera (Acevedo, 2004), sirvió de fundamento para el surgimiento de la violencia contra estatal protagonizada por los diferentes grupos guerrilleros. (Vargas, 1996)

La segunda mitad de los años sesenta presenció cierto agotamiento del pacto político y los esfuerzos de reinvención del poder ejecutivo en cabeza del Dr. Lleras Restrepo no dieron resultados. Interesado éste en propiciar una democracia más directa, tomó cierta distancia de los partidos políticos y optó por una po- 
lítica de mano fuerte hacia los sectores opuestos al sistema político. Muestra de ello fue la ilegalización de la Fundación Universitaria Nacional (FUN) y la represión a la que fueron sometidas todas las expresiones de reivindicación social y política (Leal, 1984). Recuérdese que el 24 de octubre de 1966 el presidente Lleras decidió acudir a la Universidad $\mathrm{Na-}$ cional en compañía de John Rockefeller a la inauguración de un edificio que se había construido con el apoyo del magnate estadunidense. La protesta obligó a la fuerza pública a invadir la ciudad universitaria y a detener a varias decenas de estudiantes. Para el mes de noviembre la FUN se hallaba desmantelada para luego ser declarada ilegal. Este tipo de acontecimientos arraigó más el escepticismo de los estudiantes hacia el Frente Nacional, madurando las condiciones para una oposición cada vez más evidente en las calles y en las urnas.

Por su parte, las relaciones con los actores sociales no fueron menos complicadas y azarosas. Si bien inicialmente las centrales obreras (Unión de Trabajadores de Colombia, UTC y Central de Trabajadores de Colombia, CTC) no realizaron considerables manifestaciones, ciertas medidas económicas lesivas a los intereses de los trabajadores estimularon el enfrentamiento contra el gobierno. La propuesta de capitalizar el Fondo Nacional del Ahorro con los aportes a cesantías de los obreros generó una aguda desconfianza y distanciamiento de los sindicatos y empleadores. Todo se desbordó en 1969 cuando se convocó a manifestaciones contra el alza de los precios del transporte, acciones que si bien iniciaron en Cali, terminaron por afectar a todo el país. Cabe destacar que varias de estas acciones no eran lideradas por políticos o estructuras partidistas de iz- quierda reconocidas, sino por sacerdotes cercanos a la teología de la liberación. (Restrepo, 1995)

Todas estas tensiones, impulsadas sin duda por las acciones reformistas del Dr. Lleras, pusieron de presente una situación de crisis social general. La primera en manifestar las crisis fue la Iglesia Católica. En efecto, la Iglesia era un actor que venía experimentado cambios considerables en su dinámica interna, no sólo en cuanto a la lucha por mantener sus privilegios como institución secular respecto al poder civil se refería, sino por los vientos de renovación que soplaban en su interior (Arías, 2009), Lo que se puede llamar renovación de la Iglesia para este periodo ha sido estudiado bajo la óptica del impacto del Concilio Vaticano II (1962-1965) y la II Conferencia Episcopal Latinoamericana celebrada en Medellín en 1968. La importancia de estos dos eventos para posicionar al interior de la Iglesia Católica una opción por los pobres, es reconocida en el marco de los cambios que experimentó el país y el continente en aquellos años. Las figuras de Camilo Torres en Colombia, el grupo Golconda o los Sacerdotes por América Latina (SAL) son las más reconocidas para el caso colombiano. La lectura del evangelio en clave popular y la propuesta de una Teología de la Liberación hacen parte de las rupturas que se fueron gestando en aquellos años y que tendría un interesante eco en la organización de la protesta popular y en la configuración de un campo de izquierda en el país. También el sector educativo manifestó algunos síntomas de crisis. De hecho, desde 1966 los conflictos con los estudiantes se hicieron cada vez más comunes, llegando en ocasionas a asumir ribetes violentos $\mathrm{y}$ fuertemente contestatarios. (Pecaut, 2006) 
El Frente Nacional fue entonces un periodo en el que la movilización estudiantil, pero no solo ella, como se ha visto, experimentó un súbito auge. Aunque el estudiantado nunca pudo organizarse como una sola fuerza nacional, ello no fue obstáculo para ahondar en su radicalización ideológica vinculada a las múltiples líneas de la ideología de izquierda. Desde finales de 1969 los conflictos estallaron en universidades como la Tecnológica de Pereira e Industrial de Santander, a las que se sumaron la Universidad Nacional, la Universidad del Valle y la de Antioquia e incluso algunas privadas como la Gran Colombia y la Pontificia Bolivariana en Medellín. Tal situación se complicó durante el primer trimestre de 1970, debido al cierre al que fue sometida la Universidad Nacional desde el 24 de febrero hasta el 13 de abril, hecho que generó la solidaridad de las universidades privadas más importantes de la capital. Este panorama se obscurecería aún más para el gobierno de turno debido a la convocatoria de huelga general de la central de educadores, que ya desde entonces luchaba por mejores condiciones laborales.

El último periodo presidencial del Frente Nacional da muestras del agotamiento del sistema. Como resultado de la reforma constitucional de 1968, las elecciones de 1970 para asambleas departamentales y concejos municipales no siguieron la regla de la paridad, lo que permitió la competencia política en todos los niveles político-administrativos. Este mecanismo de desmonte gradual de la nueva estructura política sirvió de ensayo para la contienda presidencial de 1970, una lucha en la que se enfrentaron liberales, conservadores, anapistas y comunistas. Aunque el vencedor fue el Dr. Misael Pastrana Borrero, un candidato con el cual el Frente Nacional lograba salir ileso, la contienda demostró que el país, o por lo menos la mayoría de la ciudadanía, ya no confiaba en el régimen. Los datos de las elecciones y la controvertida elección de Pastrana sustentan esta idea. Como se sabe, el General Rojas Pinilla denunció que en la elección de Pastrana se había cometido fraude, ya que según sus estimativos él había ganado con amplia mayoría. No obstante, los datos oficiales señalaron que Rojas Pinilla había obtenido 1.561.468 votos, mientras que el representante del FN, había ganado el favor de 1.625 .025 sufragantes (Mayorga, 2013). Poco más de 60.000 votos habían hecho la diferencia, un dato sin duda significativo, si se tenía en cuenta que la Alianza Nacional Popular (ANAPO) de Rojas era la antítesis del Frente Nacional.

Fraude o diferencia casi insignificante, los resultados electorales de 1970 dejaron en claro que el nivel de agotamiento de un amplio del electorado era alto. Es más, como la ANAPO había podido aglutinar en sus bases tanto a liberales como a conservadoras - sin contar a los diferentes grupos de izquierda que sentían afecto por el giro socialista que a veces tomaba el discurso del ex dictador-, era evidente que el Dr. Pastrana recibía la presidencia en condiciones poco favorables. Y si bien la animadversión del pueblo podía ser irrelevante para el presidente, de la resistencia anapista que encontraría en el Congreso no podría opinar igual, razón por la cual los últimos cuatro años del Frente Nacional requirieron del estado de sitio para garantizar su normal desarrollo. Así pues, como lo ha señalado Ayala (2006), el repunte de Rojas Pinilla evidenció la existencia de un ambiente social y político cada vez menos proclive al régimen bipartidista. 
Con todo, no hay que olvidar que Misael Pastrana Borrero encabezó un equipo de gobierno que se propuso no solo identificar las demandas sociales de gran parte de la población, sino de implementar soluciones plausibles. Muestra de ello fueron los intentos de reforma en varios aspectos de la vida social, económica y política, tales como la extensión de la seguridad social a las áreas rurales, la continuación de la intervención del gobierno en la economía, el mejoramiento del empleo y una reforma educativa dirigida a todo el sistema. A ello se sumaba la intención de profundizar la reforma agraria de su antecesor, aunque mejorando las relaciones con el sector privado de la economía. No obstante, y debido principalmente a que la situación en el campo se tornaba difícil, pues los programas oficiales no daban abasto, la reacción de los campesinos consistió en radicalizar su lucha. Estimulados por la idea de la expropiación se lanzaron en masa a la invasión de tierras durante el primer semestre de 1971. La Asociación Nacional de Usuarios Campesinos (ANUC), creada por Alberto Lleras con el objeto de organizar el acceso de los campesinos a los servicios del estado, progresivamente fue tomando distancia de los lineamientos oficiales para acercarse a otros movimientos sociales, como los obreros y los estudiantes, con el fin de propiciar situaciones revolucionarias. Cabe recordar que los usuarios campesinos experimentaron un proceso de politización hacia la izquierda, hecho que los llevó, a apoyar, además de las invasiones de tierra, formas de protesta cada vez más radicales, tales como la toma de oficinas públicas. La presión política que estos grupos sociales empezaban a ejercer, obligaron al gobierno a realizar una revisión de sus directrices administrati- vas, pero no para ceder a las demandas sino para contenerlas.

Con unas organizaciones campesinas cada vez más alejadas de la órbita gubernamental, una aguda situación de movilización universitaria y una fuerte tensión con los maestros y otros gremios nacionales, el Frente Nacional parecía derrumbarse. Por ello, apenas parecía lógico que el gobierno de Pastrana intentara combatir la resistencia social señalando nexos entre los manifestantes y los grupos subversivos. La agitación social que se vivió en los primeros años de la década del setenta fue de hecho asociada a la presencia de guerrilleros en las organizaciones sociales.

\section{METODOLOGÍA}

Para llevar a cabo esta investigación se acudió a la metodología del análisis histórico de perspectiva contextual, es decir, un análisis de fuentes de información primaria y secundaria que permitiera identificar los aspectos y las circunstancias que definieron la época objeto de estudio en relación con el FN. Hay que tener presente que esta metodología permite ubicar los sucesos políticos en relación con los demás aspectos de la realidad histórica, facilitando así su comprensión.

El estudio está organizado en dos apartados. En el primero de ellos se analizan las condiciones de posibilidad del $\mathrm{FN}$, es decir, las circunstancias políticas y sociales que le dieron origen. En el segundo, con la intención de ubicar los aspectos tanto positivos como negativos del pacto frente nacionalista, se describe su desenvolvimiento. De esta manera, se logra ubicar cada uno de los procesos mediante los cuales el acuerdo pudo fortalecer la 
institucionalidad, pero también aquellos aspectos que truncaron una apertura democrática que distintos sectores sociales venían exigiendo de antaño.

\section{RESULTADOS}

La crisis que vivía el país durante el último gobierno del Frente Nacional tenía una doble cara. Por una parte, la situación política no era la mejor para los partidos tradicionales, toda vez que al agotamiento del régimen paritario se le sumó la atomización partidista y la emergencia de una fuerza política cuya principal pretensión era la de erigirse en el tercer partido de la nación -la Tercera Fuerza de Rojas Pinilla-. Este desplazamiento político se fundó en cierta polarización social expresada en la votación presidencial, cuando los sectores populares se inclinaron por Rojas Pinilla, mientras que los sectores medios depositaron su voto por Pastrana. Esta realidad ponía en riesgo el carácter poli-clasista del sistema de partidos en el país y con ello al mismo Frente Nacional. Por otro lado, a nivel social, no cabe duda que las movilizaciones del estudiantado, los campesinos, los obreros, los ciudadanos de a pie o los trabajadores oficiales, radicalizados o no, lograron en ocasiones poner en jaque al sistema. La manera como procedieron los campesinos, por ejemplo, al expropiar por su cuenta grandes extensiones de tierra, promover la autogestión económica y liderar una profundización de la reforma agraria, alarmaron tanto al gobierno, que lo obligaron, con la aquiescencia, o más bien con la demanda de los terratenientes y los gremios, a poner fin a la reforma agraria. Así pues, cercanos-o influenciados- a las diferentes corrientes de la izquierda, estos actores sociales reprodujeron de acuerdo a sus especifici- dades las tensiones ideológicas y políticas de toda una época.

El 16 de abril de 1972 se llevaron a cabo las elecciones para los cargos locales y departamentales. En un contexto social caracterizado por la crisis, era obvio que el panorama político apenas se modificara. Lo que no parecía evidente, sin embargo, era que el bipartidismo nuevamente saliera indemne. Los partidos tradicionales continuaron sus pugnas internas, pero le dieron a Pastrana un margen de maniobra adecuado para que terminara su periodo presidencial. En las filas liberales se planteó un duro enfrentamiento entre el ala de los turbayistas (seguidores de Julio C. Turbay) y el ala de los lopistas (seguidores de Alfonso López). En las toldas conservadoras, por su parte, la lucha enfrentó a los ospinistas (seguidores de Mariano Ospina), contra los alvaristas (seguidores de Álvaro Gómez). La izquierda saltaba al ruedo con el Movimiento Obrero Independiente Revolucionario (MOIR). El gran derrotado era el movimiento del General Rojas Pinilla. Tal como había ocurrido en 1957, quince años después -ahora al comando de un nuevo partido, y tal vez debido a que la dirigencia anapista no se pudo sintonizar con la radicalización de sus bases, o a que en varios municipios actuó como cuota burocrática- el grupo de Rojas se desgastaba políticamente. Así pues, desactivada la amenaza anapista, el Frente Nacional confirmó, en su último gobierno, que la fidelidad bipartidista seguía viva, y que si bien ya no parecía necesario mantener un pacto de unidad nacional, en lo que quedaba del siglo XX un reordenamiento político bipartidista seguiría primando, ahora, por su puesto, en el marco de una nueva estrategia económica mundial. (Pecaut 2006) 


\section{CONCLUSIONES}

La principal consecuencia del FN consistió en cerrar el camino de la contienda política a otros sectores sociales; de hecho, en vez de "ampliar el espectro del sistema de partidos", propendió por la "absorción de los programas de los movimientos de oposición", de manera que el país "desaprovechó la conformación de un sistema de partidos diversificado que habría servido para jalonar el desarrollo político del país". La historia habría sido otra si grupos como el Movimiento Revolucionario Liberal, la Alianza Nacional Popular, el Movimiento Democrático Nacional, la Democracia Cristiana, el Frente Unido, el Partido Comunista y las dos organizaciones guerrilleras nacidas en esta época -el Ejército de Liberación Nacional (ELN) y las Fuerzas Armadas Revolucionarias de Colombia (FARC) hubieran tenido abiertas las puertas de la participación política. Sin duda, el Movimiento 19 de Abril (M-19) hubiera carecido de una justificación histórica si los defensores del Frente Nacional no le hubiesen cerrado las puertas de la participación política a su principal grupo opositor, la Alianza Nacional Popular. (Báez, 2006)

\section{REFERENCIAS}

Acevedo, Á. (2004). El símbolo de un Robin Hood vengado en el occidente de Colombia. Revista Estudios Humanísticos-Historia, (3), 45-66.

Archila, M. (2003). Idas y venidas, vueltas y revueltas: revueltas y protestas sociales en Colombia, 1958-1990. Bogotá: ICANH-CINEP.
Arías, R. (2009). El episcopado colombiano en los años 1960. Revista de Estudios Sociales, (33), 95-158.

Ayala, C. (1999). Credencial Historia. Recuperado el 31 de Enero de 2014, de Frente Nacional: acuerdo bipartidista y alternación en el poder: http://www.banrepcultural.org/ blaavirtual/revistas/credencial/julio2012/indice

Ayala, C. (2006). El populismo atrapado, la memoria y el miedo: el caso de las elecciones de 1970. Medellín: La Carreta/Universidad Nacional de Colombia.

Báez, A. (2006). La Alianza Nacional Popular (Anapo) en Santander, 19621976. Bucaramanga: Universidad Industrial de Santander.

Berry, A. (2012). La tragediade la reforma agraria del Frente Nacional. En C. Caballero, M. Pachón Buitrago, \& E. Posada Carbó, Cincuenta años de regreso a la democracia: nuevas miradas a la relevancia histórica del Frente Nacional (págs. 295-323). Bogotá: Uniandes/Escuela de gobierno Alberto Lleras Camargo.

Calle, H. (2012). El plebiscito de 1957, la legitimidad fundacional del Frente Nacional. En C. C. otros, Cincuenta años de regreso a la democracia: nuevas miradas a la relevancia histórica del Frente Nacional (págs. 119-146). Bogotá: Uniandes/Escuela de Gobierno Alberto Lleras Camargo.

Colombia, Congreso de la República de (1961). Ley 135 Sobre reforma social agraria. Bogotá DC: Diario Oficial 30691 
Hartlyn, J. (1993). La politica del Régimen de coalición: La experiencia del Frente Nacional en Colombia. Bogotá: Centro de Estudios Internacionales de la Universidad de los Andes (CEI)/Uniandes/Tercer Mundo Editores.

Henderson, J. (1984). Cuando Colombia se desangró: un estudio de la violencia en metrópoli y provincia. Bogotá: El Áncora.

Henderson, J. (2006). La modernización en Colombia: los años de Laureano Gómez, 1889-1965. Medellín: Universidad de Antioquia.

Junguito, R. \& Rincón, F. (2007). La política fiscal en el siglo XX en Colombia. En J. Robinson, \& M. Urrutia, Economía colombiana del siglo XX: un análisis cuantitativo (págs. 239-308). Bogotá: Fondo de Cultura Económica.

Kalmanovitz, S. (1995). Economía y nación, una breve historia de Colombia. Bogotá: Tercer Mundo.

Leal, F. (1984). La participación política de la juventud universitaria como expresión de clase. En M. Cárdenas, \& A. Díaz Uribe, Juventud y politica en Colombia (págs. 155-203). Bogotá: FESCOL/Instituto SER.

Machado, A. (1998). La cuestión agraria en Colombia a fines del milenio. Bogotá: El Áncora.

Machado, A. (2011). Centro de Estudios Estadounidenses. Recuperado de: http://www.ceecolombia. org/ckfinder/userfiles/files/La\%20 alianza $\% 20$ para $\% 20$ el $\% 20$ pro greso $\% 20 y \% 201 \mathrm{a} \% 20$ reforma $\% 20$ agraria $\% 20($ Absal\%C3\%B3n $\% 20$ Machado).pdf

Mayorga, F. (2013). Historia de la organización electoral en Colombia (1888-2012): vicisitudes de la consolidación democrática en un país complejo. Bogotá: Universidad del Rosario/Facultad de Jurisprudencia.

Musgrave, R. (1969). Informe Musgrave: bases para una Reforma Tributaria en Colombia. Bogotá: Biblioteca Banco Popular.

Palacios, M. (1998). Entre la legitimidad y la violencia, Colombia, 1875 1994. Bogotá: Norma.

Palacios, M. (2012). Violencia pública en Colombia, 1958-2010. Bogotá: Fondo de Cultura Económica.

Pecaut, D. (2006). Crónica de cuatro décadas de política colombiana. Bogotá: Norma.

Plazas, M. (2011). El Frente Nacional. Bogotá: Temis.

Ramírez, M. \& Tellez, J. (2007). La educación primaria y secundaria en Colombia en el siglo XX. En J. Robinson, \& M. Urrutia, Economía colombiana del siglo $X X$ : un análisis cuantitativo (págs. 459-513). Bogotá: Fondo de Cultura Económica.

Restrepo, J. D. (1995). La revolución de las sotanas: Golconda 25 años después. Bogotá: Planeta.

Tirado, Á. (1981). Colombia: siglo y medio de bipartidismo. En M. Arrubla, Colombia hoy (págs. 100-189). Bogotá: Siglo XXI. 
Urrutia, M. (2012). Política económica y social en el régimen de coalición. En Caballero, \& C. y. Caballero, Cincuenta años de regreso a la democracia: nuevas miradas a la relevancia histórica del Frente Nacional (págs. 241-258). Bogotá: Uniandes/Escuela de gobierno Alberto Lleras Camargo.
Vargas, A. (1996). Política y armas al inicio del Frente Nacional. Bogotá: Universidad Nacional de Colombia. 


\title{
THE NATIONAL FRONT: INSTITUTIONAL LEGITIMACY AND BIPARTISAN CONTINUISM IN COLOMBIA (1958-1974)'
}

\author{
Álvaro Acevedo Tarazona ${ }^{2}$ \\ Universidad Industrial de Santander, Colombia - Type 1 Article. \\ Scientific and Technological Research-Received: April 10th 2015 - Accepted: June 13 th 2015 \\ doi: http://dx.doi.org/10.17981/econcuc.36.1.2015.22
}

\begin{abstract}
Through a contextual historical analysis, an approach that allows the reconstruction of the main social, political, cultural and economic characteristics of a period of time; this paper has its focus on the elements that made possible the emergence of the National Front as a two-party agreement, and analyzes the actions that contributed to the emergence of new obstacles for the development of democracy in Colombia. While the violence in the 1950s and the dictatorship of General Gustavo Rojas Pinilla were identified as causes of the emergence of the new political structure of the country, the political exclusion of unprecedented social actors such as the peasant movement, or more recent ones such as the labor movement and the student movement, was perceived as its main consequence. As a result of the analysis, this study allowed the configuration of a new hypothesis on the historical meaning of the National Front, concluding that, however the National Front helped to strengthen the political and economic institutions of the country, when legalizing the discrimination of any other social force that intended to represent civic interests the agreement favored the appearance of a new historical problem for Colombian democracy: the exclusion of opposition political actors, a facet that is still in force in this democracy.
\end{abstract}

\section{Keywords:}

National Front, Dictatorship, Plebiscite.

JEL: D74, D78, F52

\section{If you need to reference this article}

Acevedo, A. (2015). The National Front: Institutional legitimacy and bipartisan continuism in Colombia (1958-1974), Económicas CUC, 36(1), 49-78. doi: http://dx.doi.org/10.17981/econcuc.36.1.2015.22

\footnotetext{
1 This paper is the product of the research project: "The historical experience of "co-government" at Universidad Industrial de Santander: conceptions and divergences in dispute over university autonomy, 1971-1976", financed by the Office of the Vice Chancellor for Research from Universidad Industrial de Santander.

${ }^{2} \mathrm{PhD}$ in History from Universidad de Huelva. Permanent professor at Universidad Industrial de Santander, Bucaramanga. Head of the Research Group "Políticas, Sociabilidades y Representaciones Histórico-Educativas (PSORHE)", acetara@uis.edu.co
} 


\section{INTRODUCTION}

In this paper, the history of the National Front (NF) (Frente Nacional, FN), the political agreement under which the country lived between 1958 and 1974, is described and analyzed. The analysis deals with its origin, as well as with its development and legacy. Not only its legal origin, through the 1957's plebiscite, is examined, but also both the social and political circumstances that set the context where it appeared as an inevitable response to the political violence of the mid-twentieth century, and the dictatorship of General Gustavo Rojas Pinilla. Although it is widely recognized that the NF was a strategy that allowed the strengthening of the political and economic institutions in Colombia, it is important to have present that its modus operandi -characterized by the legal recognition of the two traditional political forces: Liberal and Conservative parties- originated new and urgent national problems, not only for legalizing the discrimination of the political actors that did not identify with the traditional parties, but by fighting any force that intended to gain the society's political recognition. In fact, as it is described in this paper, the NF allowed to restrain the violence and redirect the country to the path of democracy, but it left out of its reach a series of aspects that deserved its attention, such as the opening of new ways of political participation for the social sectors that the economic development was "shoring up".

This paper attempts to argue, consequently, that although the NF marked the political life of the country, it was not only because it evidently reduced the political violence that was "bleeding" the country dry, but because it also managed to exclude and defeat the political forces that intended to emerge as the new representatives of the nation, and all of this through its main formula: the alternation and parity of the traditional parties. Indeed, the student move- ment, the labor movement struggle and the organization of new political groups, influenced undoubtedly by what was happening around the world, but also driven by their own forces; tried unsuccessfully to gain political and social visibility during the NF, even by joining a populist leader such as Rojas Pinilla, who was the same that the official two-party system had replaced in order to recover democracy.

\section{DEVELOPMENT}

\section{The conditions allowing the possibility of the National Front}

Colombia has not been the only country to resort to bipartisan agreements in order to give a solution to their problems related to institutions and state weakness. Uruguay and Venezuela in the 1950s and 1960s, and Chile and Argentina in the 1980s, only mentioning some cases in Latin America, experienced something similar during the periods of transition or reconquest of their democracies. In order to analyze these types of facts and to understand the Colombian case, Hartlyn (1993), used a concept from political science, consociationalism $^{3}$, understanding this as the political phenomenon whereby traditional ruling classes make coalitions that facilitate the transition toward a democratic government, but restrict democracy itself in a certain way. Yet, this phenomenon should not be thought to be the same in all countries. Contrarily, it must be recognized that in every country, given its political peculiarities, consociationalism is developed in a different way. Therefore, the first question that we need to try to solve in order to understand this stage of our recent history is

\footnotetext{
3 Consociationalism generally refers to a form of government recommended for the management of conflictsin deeply divided societies. It is often considered as a synonym of power-sharing.
} 
which were the facts that led to the establishment of that National Front agreement as a way of transition toward democracy in Colombia?

Without a doubt, a set of complex historical circumstances turned the National Front agreement into a necessity. Indeed, the violence and the dictatorship of General Rojas Pinilla had sunk the country in a deep well of illegitimacy. Consequently, agreeing on a new political order became inevitable. It was for this reason that having the society to decide on a conciliatory political project was proposed, since only through a plebiscite, as explained by Calle (2012), the nation would be able to "recover the constitutional thread" after The Violence, and the dictatorship would have lost.

The Violence with a capital V, conceived as a bloody period of time in Colombian history when liberals and conservatives "swept away" the country with reasons no different to hate itself; as it is known, was triggered by the murder of Jorge Eliécer Gaitán, on April 9 ${ }^{\text {th }} 1948$. Certainly, it was that event that made the deep gap that separated the two political elites and ordinary people visible. “Gaitán's shooting -Henderson tells us (2006, p.452)- threwhis followers into a frenzy" of revenge. Hate, just as fire, had been rekindled.

These facts were the prelude to a civil war that after 1949 will only know brief and unjustifiable lapses of bipartisan peace, since the general scene would be dominated by intransigenceand mutual enmity, until it ended in no less than the discontinuation of the democratic government. Around June1949 liberals presented to the legislative elections with the security that they would be a majority in the Congress, and that they would offer strong opposition to the conservative administration of Dr. Mariano Ospina Pérez, whom they blamed for The Violence, as they considered him weak and lacking the necessary leadership to stop the people from bleeding. However, and even though they were the majority of the legislative branch, the strategy did not work since the political constitution enabled the executive power to dissolve the Congress in the case that public order was disrupted. It was precisely this what Ospina did on November9 ${ }^{\text {th }} 1949$ : declaring a state of siege and suspending the Congress. In the face of such an action, liberals considered that they only had one last way out: creating guerrilla groups in order to get by force what they had not achieved through political means.

From that moment, resorting here to the concept recently proposed by Palacios (2012, p.25), there is no doubt that Colombia was immersed in the first -and one of the worst- waves of "public violence", i.e., of the type of violence, both social and by the state, that requires "a self-legitimation discourse" to justify the "tragedy of thousands of homes and neighborhoods", the violation of "the moral codes" and the curtailment of the social bonds of the country.

In any case, even though the behavior of the phenomenon of violence was dissimilar, there is no doubt that it kept the whole country in a state of maximum alert. In different regions, but mainly in the Llanos Orientales and Zona Cafetera, territories that the government could barely reach with its military force, the gangs and guerrillas, at the beginning of liberal origins, and sometime later communist, began to be present, to control the situation and to run rampant eliminating their opponents and constraining the popular will during the electoral periods (Henderson, 1984). But in addition to these facts, clearly truthful, the country also started to experience the myth of The Violence. In fact, Palacios (1998), explains that the episodes of violence taking place between 1945 and 1964 became, due to oral tradition, a "collec- 
tion of more or less truthful testimonies, transformed into fragmentary legends by the countless succession of narrators. The 1950s and 1960s encouraged a series of essays, novels, theater performances, cinematographic productions and visual art creations" that spread the 'black legend' of The Violence in Colombia.

Having said that, in addition to these facts there was a darker and darker political scene. In 1950, after declaring the state of siege and after the elections, Dr. Ospina Pérez handed the power to the conservative Dr. Laureano Gómez. This last had set himself the goal of averting violence through a constitutional reform that reconsidered, among other aspects, the functions of legislative power, restored the prevailing role of the Catholic Church in the management of education, limited the freedom of expression, and removed from the Congress the faculty to legislate over the functions of the armed forces. Although the reform always remained a project, it was not well received by liberalism, and even a sector of conservatism were also leery of it. Therefore, from an early stage Gómez's administration earned the abhorrence both from liberals and from their own supporters. Laureano Gómez, Palacios (1998, p.210211) explains, "did not understand that the limits of his authoritarianism were set beforehand", and that "his administration excluded too many interests", mainly those of Armed Forces, so he did not see the putsch that put an end to his administration coming. And in fact, "the night of June $13^{\text {th }}$ 1953, and with the support of Dr. Ospina himself and the leaders of the conservative opposition, General Rojas Pinilla announced the consummation of a coup d'état. The Catholic Church, entrepreneurs, and all political groups, with the exception of a handful of laureanistas and supporters of the communist party, endorsed it". (Palacios, 1998, p.211)
The military uprising was received with consent by several political sectors; some of them even received Rojas as the savior of the nation (Tirado, 1981). In order to show more signs of legitimacy, Rojas and his followers organized a National Constituent Assembly with the objective of declaring, on the one hand, that on June $13^{\text {th }}$ the presidency had become vacant, and on the other, that Rojas would take the post until the institutions were reorganized. His administration was marked by the following features: as soon as he rose to have the power and with the purpose of assuring his reelection, the general started a marathon proselytizing tour around the country; he formed a government staff that included, not in an equitable way though, liberals and conservatives, being the latter the most numerous; he solved the Los Llanos conflict, by reducing the number of deaths due to violence from 22,000 in 1952-1953 to 1,900 in $1954-1955$, and attacked judicial corruption through the dismissal of employees that were supporters of Laureanismo. (Henderson, 2006)

In April 1954, Gustavo Rojas Pinilla summoned one more time the National Constituent Assembly, which had been dissolved in June 1953, after legalizing Rojas's ascend to power. The objective of the new summons was announcing that the country did not have the public order conditions to conduct the presidential elections, as well as declaring that he committed to leave his position as soon as the order returned. Meanwhile, Rojas introduced women's suffrage, constitutionally proscribed the communist party, and replaced Departmental Assemblies and Municipal Councils by different administrative bodies. In August 1954, the National Constituent Assembly extended Rojas's term until 1958. For liberals this had been Rojas's most daring political move, and from their combat platforms, El Tiempo and El Espectador news- 
papers, they decided to restrain it. The NF responded ordering the closing of the newspapers. It was in that moment when the military dictatorship became evident and, although under the "aegis" of General Rojas the country was experiencing better times -the coffee boom and the financial stability were a proof of that, as shown by Kalmanovitz (1995)-,no elaborated reasons were necessary to argue that the extension meant a greater undermining of democracy.

The traditional parties did not hesitate then to remove from him the military support they bestowed in 1953. Nor did they hesitate to echo the requests that a great part of society was making at least since 1954, with regard to the freedom of expression and mobilization. Nevertheless, the straw that broke the camel's back was the clear manifestation of force that General Rojas Pinilla made on June $13^{\text {th }} 1956$, at the capital city's football stadium, when he presented the political party he had founded, called Third Force (Tercera Fuerza), to the crowd. The event was a complete ritual, since "thousands of participants" marched "before the "Supreme Leader»", dressed in "all his elegance and decorations", as they swore before God that they would be loyal to the people and the armed forces (Palacios, 1998, p.215-216). If to such event we add-as gems-the murder of a student from Universidad Nacional on June $8^{\text {th }} 1954$ by Colombia battalion, or the imposition of a tax to the benefits of industrial people, events that both students and unions rejected, there was no doubt that the controlling class needed a new way out. Therefore, as explained by Tirado (1981), the leaders of the two traditional parties did not have any other choice than reaching an agreement and joining forces to fight their new common enemy. Rojas's dictatorship was, consequently, the second of the contextual elements that would make the emergence of the National Front possible.

\section{The development of the National Front}

The plebiscite that brought the National Front to life took place on the first Monday of December 1957.That day, for the first time in the republican history of Colombia, women massively attended the polls. Toward the end of the journey former President Alberto Lleras Camargo confirmed that the document of the agreement between parties had been endorsed by the $94.8 \%$ of voters, that is, by $4,169,294$ votes. Only the $4.7 \%$ of citizens voted against it. If it is taken into account, additionally, that the $81.95 \%$ of the electoral register participated, there is no doubt that the National Front, in fact, got the democratic legitimacy that the parties expected. (Calle, 2012)

What did Colombians vote for? The document of the agreement indicated the following (Plazas, 2011):

1. It gave women the same political rights that men already had.

2. It established the parity of posts, both of popular election and administrative appointment, for liberal and conservative parties.

3. It indicated that, in order to resolve any problems at any corporation, the majority was considered to be two of three parts of the whole.

4. It acknowledged the President' sright to freely appoint and remove the members of his cabinet, but forced him to give participation to both political parties in the same proportion that they had in the legislative chambers.

5. It gave the Congress of the Republic the faculty to establish and regulate the functions and guarantees of government employees. 
6. It prohibited that the government employees participated in proselytizing activities, without detriment to the free practice of their right to vote.

7. It noted that under no circumstances the political affiliation of citizens could determine their appointment in a government employment or position, or their discharge or promotion.

8. It noted that the members of the Congress and of the Department Assemblies would not have a permanent salary, but daily wages during the period of sessions; and

9. It established that the government would invest $10 \%$ of its national budget in public education. It ordered that constitutional reforms could only be made by the Congress.

After winning the elections on May $4^{\text {th }}$ 1958, Dr. Alberto Lleras Camargo received a country that was still battered by violence. Even though the rates of homicides did not reach the peaks from its first stage (1947-1953), the conflict still sacrificed thousands of Colombians (Henderson 2006, Annex 2).Hence, in order to accelerate the process of pacification of the country, the new president decided, on the one hand, to maintain the program of amnesty and pardon that the Military Government had established; and, on the other, to initiate a committee of rehabilitation of the victims of violence. His efforts had a relative success. A year later, the reduction of deaths due to political violence was significant -a third less than the previous year- but this indicated that there were still a little less than 2,000 deaths per year.
In fact, the country was starting to experience new and serious socio-economic problems, such as the population growth, the currency crisis, the fiscal deficit, and the social inequality due to the concentration of lands. With regard to the population growth, the following can be noted. It was a global phenomenon that affected both the countryside and the city, and that alarmed not only the local elites but the foreign experts that were advising the new administrations. Dr. Lleras Camargo himself considered that the country was not prepared for the design of policies to contain the rapid population growth. Consequently, since the Catholic Church and the conservative party exerted a strong pressure to stop the government from introducing and directing modern programs for birth control, the National Front administrations had to leave the application of modern technologies for birth control to private entities. This move, whether recognized or not, worked well, since, ultimately, it stopped the program from being politicized, and let it reached the great majority of the population. (Urrutia, 2012)

The currency crisis, on its hand, beginning in 1957, could barely be contained around 1960, since the phenomenon quickly acquired a structural and longterm character. Since the import of industrial and agricultural supplies was needed for the industrialization policy, and due to the fact that the export of raw material was growing at a lower rate, the balance of payments was negative all of the time. The phenomenon, common to almost all Latin America, led countries like Colombia to adopt the two-gap model, an economic concept of the time where underprivileged countries had to, on the one hand, increase their exports, and on the other, gain more international help, as it was thought that the main problems of 
developing countries originated from the shortage of foreign currency and savings. As it may be supposed, the two-gap model marked the economic policy of the whole period, forcing the National Front administrations not only to focus their administrative efforts on the search of external credit, but also on the organization of their own budgets according to the interests of the banks that accepted to inject foreign currency in the countries in need of them. (Kalmanovitz, 1995)

But not all of it was negative, the bipartisan agreement, with the threats of left-wing totalitarianism pervading Latin American atmosphere, helped Colombia to gain the support of the U.S.A. Certainly, in the period of the National Front foreign help constantly flowed toward Colombia, primarily from the administration of President Kennedy, who, with "the intention of repeating the success they had had with the Marshall Plan'in Europe", created the Alliance for Progress for Latin America. External help became, consequently, a core element of the economic and social politics of the National Front administrations, initially because it was the only way to gain access to soft, long-term credits, and ultimately because it was impossible to think of other ways to boost the country's development and reinforce its economic structure, given that without the guarantee of international agencies such as the International Monetary Fund and the World Bank-Colombian economy would not have reached a certain degree of stability. (Urrutia, 2012, p. 247)

\footnotetext{
${ }^{4}$ The officially European Recovery Program or ERP was the most important plan of the United States for the reconstruction of European countries after the Second World War, which was at the same time intended to prevent the possible spread of communism. The initiative was named after the U.S. Secretary of State, George Marshall, and was designed mainly by the State Department, particularly by William L. Clayton and George F. Kennan.
}

Nevertheless, the country never stopped experiencing the ups and downs that are typical of the market economy. Indeed, that macroeconomic policy suffered several and periodic currency mismatches, due mainly to the fact that Colombia was keeping inflation rates higher than international ones. This situation forced the governments to "make periodic nominal devaluations", which brought high political costs, as it was necessary to adjust the price of products that had an important role in economy, such as imported food, gas and transportation. Hence, to a very large extent -as Archila's study (2003), on protest in Colombia has proven- there were many manifestations led by civil society to demand the removal of those strictly economic government regulations that were ultimately detrimental for ordinary citizens.

The third problem that the National Front governments were forced to face was the constant fiscal deficit. Certainly, during the whole period the country experienced a strong fiscal restriction. Around 1969, the Musgrave report noted that the country's public purse was barely an $8 \%$ of the gross domestic product, even though the administrations of Dr. Lleras Camargo and Dr. Guillermo León Valencia had initiated and reinforced a tax reform (Musgrave, 1969). The social difficulty that this brought was related to the impossibility of the State to take responsibility for the supply of public goods and services, as well as of the required subsidy for the productive sector (Junguito \& Rincón, 2007). The shortage of tax resources increased the dependency on external credit, and since this only supported investments in infrastructure, the service sector was left practically beyond recovery. Only the education sector, undoubtedly because the plebiscite ordered so, would count on a higher investment. Therefore, during the National Front, public investment in- 
creased from 1\% of the GDP between 1938 and 1958 , to $3.5 \%$ in the early $1960 \mathrm{~s}$. (Ramírez \&Tellez, 2007)

The National Front governments also had to deal with serious problems of social inequality due to the concentration of lands. Therefore, since the initiation of the front once again in Colombian history the political, corporate and agrarian elites agreed to seriously discuss the agrarian reform -the topic had been already discussed during the liberal republic-. Around the 1960s, for the majority of politicians-among them the liberals-it was an indisputable truth that in order to accelerate its economic development the country required an agrarian reform. Back in 1951, the World Bank "had already held that the land of the latifundia was wrongly used" and that "an agrarian reform would not only improve the distribution of the income, but would also accelerate the growth of agricultural production" (Urrutia, 2012, p.249). Colombia, as many other countries, was characterized -and is still characterized- by a high concentration of land, and because of this it had very high levels of inequality. Therefore, although this is an option that is not very often used -for obvious reasons, since it was the leading class who owned the land-, the agrarian reform was considered an appropriate tool for repairing such inequality. However, as has been shown by Berry (2012, p.296) "the objectives proposed during [the National Front] were not achieved".

To summarize, experts say, the National Front found in the agrarian reform the solution "to all the problems suffered by the country during the twentieth century". However, its inappropriate and weak enforcement constituted its greatest failure (Berry, 2012, p.296). The initiative sought both to redistribute the land and to foster the industrialization of the agricultural sector. In order to promote this initiative, Dr. Lleras Camargo signed Law 135 of 1961, whereby the Colombian Institute of Agrarian Reform (INCORA), a body which would be responsible for the titling of public lands and the search of appropriate mechanisms to make Colombian agriculture more productive, was created. The law specifically forced the landowners, under penalty of forfeiture, to modernize the forms of land use, and to make a more adequate use of it (Machado, 1998). However, neither the one nor the other produced lasting effects since the implementation of the law was always hampered by the greatest landowners. During Dr. Valencia's government, for instance, while the agrarian reform was not entirely suspended, it experienced a sharp slowdown. With the victory of Carlos Lleras Restrepo, who had supported the law from the Congress during the administration of Dr. Lleras Camargo, the measure gained vitality once again. In this occasion, the state was concerned with organizing the Colombian peasantry through Decree 755 of 1967 , ordering to make a record of all peasants that were users of the state services, so that they could have reliable information about the size of this social sector.

Yet, with the return of conservatives to the power in 1970, the norm, and with it the deep illusion of Colombian peasants to achieve greater equality and social inclusion, found its end. Indeed, although for this time the process of agricultural modernization had begun, before the period of Dr. Misael Pastrana ended, the government and great landowners agreed to definitely abandon the reform (Berry, 2012). In conclusion, throughout the period of the National Front the "reform process was hampered by political, economic and legal factors, and by others of operational and technical nature" that made it unfeasible. (Machado, 2011) 
It is important to describe the main events that the new social actors experienced during that period: the student and political groups that the NF left out of the political game by ignoring the opposition (Ayala, 1999). After Gustavo Rojas Pinilla's fall and during the transition toward the two-party "system", Colombian society saw university students emerge with a considerable force. As has been noted, the NF and its parity bureaucratic structure, despite allowing the reduction and eradication of bipartisan and then organized violence (Acevedo, 2004), laid the groundwork for the emergence of violence against the state led by different guerrilla groups. (Vargas, 1996)

The second half of the sixties witnessed a certain depletion of the political agreement, and the efforts to reinvent the executive branch led by Dr. Lleras Restrepo gave no results. Interested in pursuing a more direct democracy, he took some distance from the political parties, and opted for a strong hand against the sectors opposing the political system. A proof of this was the banning of the $\mathrm{Na}$ tional University Foundation (Fundación Universitaria Nacional, FUN) and the repression that all manifestations of social and political demand suffered (Leal, 1984). It is worth to remember that on October $24^{\text {th }} 1966$ President Lleras decided to go to the Universidad Nacional (National University) accompanied by John Rockefeller for the opening of a building which had been built with the support of the American tycoon. The protest forced the police to invade the campus and arrest dozens of students. By November, the FUN was dismantled before being outlawed. Such events rooted the skepticism of students regarding the National Front, and prepared the conditions for an opposition that became more and more evident on the streets and at the polls.
Meanwhile, the relations with the social actors were no less complicated and hazardous. While initially the labor unions (Unión de Trabajadores de Colombia, UTC and Central de Trabajadores de Colombia, CTC) made no significant demonstrations, certain economic measures that were harmful for the interests of workers encouraged the confrontation against the government. The proposal to capitalize the National Savings Fund with the contributions of the severance pays of workers caused a deep mistrust and estrangement of unions and employers. It all went out of control in 1969 when demonstrations against the rising prices of transport were organized, actions that though initiated in Cali, ended up affecting the entire country. It is worth noting that several of these actions were not led by recognized politicians or left-wing party organizations, but by priests that were kin to liberation theology. (Restrepo, 1995)

All these tensions, undoubtedly fueled by Dr. Lleras' reformist actions, evidenced a state of general social crisis. The Catholic Church was the first to put the crisis forth. Indeed, this Church was an actor which had been experiencing significant changes in its internal dynamics, not only in terms of the struggle to maintain their privileges as a secular institution with regard to the civil power, but of the winds of change blowing within it (Arias, 2009). What can be called the renewal of the Church for this period has been studied from the perspective of the impact of the Second Vatican Council (1962-1965) and the Second Latin American Episcopal Council celebrated in Medellin in 1968. The importance of these two events for the positioning of an option for the underprivileged in the Catholic Church, is recognized as part of the changes experienced by the country and the continent at that time. Leading figures such as Camilo Torres, the Golconda 
group or Priests for Latin America (SAL) are the most recognized in the Colombian case. The reading of the gospel in popular language and the proposal of a liberation theology are part of the breaks that were developing in those years and that would have an interesting echo in the organization of popular protests and in the configuration of a left wing in the country. The education sector also showed some signs of crisis. In fact, since 1966 conflicts with students became increasingly common, occasionally showing violent and rebellious features. (Pecaut, 2006)

The National Front was then a period when the student movement, but not only this, as we have seen, experienced a sudden boom. Although students were never able to organize as a single national force, this did not prevent the deepening of their ideological radicalization linked to multiple lines of left-wing ideology. From late 1969 the conflicts broke out in universities such as the Tecnológica de Pereira and the Industrial de Santander, joined by the Universidad Nacional, University del Valle and Universidad de Antioquia, and even by some private institutions such as the Gran Colombia and the Pontificia Bolivariana in Medellin. This situation became worst during the first quarter of 1970 , due to the closure of the Universidad Nacional from February $24^{\text {th }}$ to April $13^{\text {th }}$, a fact that deserved the solidarity of the most recognized private universities in the capital. This scene would later darken some more for the ruling government due to the general strike call by the central of educators that, from that moment, was fighting for better working conditions.

The last presidential term of the National Front was showing signs of depletion of the system. As a result of the constitutional reform of 1968, the 1970 elections for municipal councils and department assemblies did not follow the rule of parity, allowing political competition in all political and administrative levels. This mechanism of gradual clearing of the new political structure served as a rehearsal for the presidential race of 1970 , a struggle where liberals, conservatives, communists and ANAPO (National Popular Alliance) supporters clashed. Although the winner was Dr. Misael Pastrana Borrero, a candidate that allowed the National Front to remain unaffected, the dispute showed that the country, or at least the majority of citizens no longer trusted the regime. The data of the elections and the controversial election of Pastrana support this idea. As it is known, General Rojas Pinilla claimed that there had been fraud in Pastrana's election, since according to his estimates he had won with a large majority. However, the official data showed that Rojas Pinilla had obtained 1,561,468 votes, while the NF representative won the favor of 1,625,025 voters (Mayorga, 2013). Just over 60,000 votes had made a difference, an undoubtedly significant fact, if considered that Rojas's National Popular Alliance (ANAPO) was the antithesis of the National Front.

Be it a fraud or an almost insignificant difference, the election results of 1970 made clear that the level of depletion of a great part of the electorate was high. Moreover, as the ANAPO had been able to bring together both liberals and conservatives -without counting the various left- wing groups who felt affection for the socialist twist that the former dictator's speech sometimes took-, it was evident that Dr. Pastrana received the presidency in unfavorable conditions. And while the animosity of the people could be irrelevant to the President, the same could not be said about the anapista resistance that he found in the Congress, which was the reason why the last four years of the National Front administration required the state of siege in order to ensure its normal devel- 
opment. Thus, as noted by Ayala (2006), Rojas Pinilla's recovery showed the existence of a social and political scene that was less and less prone to the two-party system.

Yet, we must not forget that Misael Pastrana Borrero led a government team that decided not only to identify the social demands of a great part of the population, but to implement plausible solutions. A proof of this were the attempts to reform various aspects of social, economic and political life, such as the extension of social security to rural areas, the continuation of the government's intervention in the economy, the improvement of employment, and an educational reform to the entire system. Together with this, there was the intention to go further with the agrarian reform of his predecessor, although improving the relations with the private sector of the economy. However, primarily because the situation in the countryside was becoming difficult as the government programs were not sufficient, the peasants' reaction was to radicalize their struggle. Stimulated by the idea of expropriation, they massively turned to land invasions during the first half of 1971.The National Association of Peasant Land Users (Asociación Nacional de Usuarios Campesinos, ANUC), created by Alberto Lleras in order to organize the access of peasants to the state services, gradually moved away from its official guidelines and approached other social movements, such as the labor and student ones, in order to promote revolutionary situations. It is worth to recall that peasant land users experienced a process of left-wing politicization, and that this led them to support, besides land invasions, increasingly radical forms of protests, such as the occupation of public offices. The political pressure that these social groups began to exert, forced the government to review its administrative guidelines, but not in order to give in to the demands, but to contain them.

With the peasant organizations more and more distant from the government's interests, a serious situation of university mobilization, and a strong tension with teachers and other national unions, the National Front seemed to be collapsing. Therefore, it was just logical that Pastrana's administration tried to combat social resistance by indicating links between demonstrators and subversive groups. The social agitation that took place in the early seventies was indeed associated with the presence of guerrillas within social organizations.

\section{METHODOLOGY}

For conducting this research, the contextual historical analysis methodology, that is, an analysis of the sources of primary and secondary information to identify the aspects and circumstances that defined the period under study in relation to the $\mathrm{NF}$, was used. It is important to keep in mind that this methodology allows to identify the political events in relation to the other aspects of historical reality, thus facilitating their understanding.

The study is divided into two sections. In the first of them the conditions allowing the possibility of the NF, that is, the political and social circumstances that gave rise to it, are analyzed. In the second, with the intention of identifying both positive and negative features of the $\mathrm{Na}$ tional Front agreement, its development is described. In this way, it is possible to identify each one of the processes whereby the agreement strengthened the institutions, but also those aspects that hampered a democratic opening that different social sectors had been demanding for a long time. 


\section{RESULTS}

The crisis affecting the country during the final government of the National Front had two sides. On the one hand, the political situation was not the best for the traditional parties, since in addition to the depletion of the bipartisan regime, there appeared the fragmentation of the parties and the emergence of a political force whose main aim was to establish as the nation's third party -Rojas Pinillas's Third Force. This political shift was originated by certain social polarization expressed in the presidential election, when the popular sectors were in favor of Rojas Pinilla, while the middle class voted for Pastrana. This situation threatened the poly-class character of the country's party system and thus the National Front itself. On the other side, on the social level, there is no doubt that the demonstrations of students, peasants, workers, ordinary citizens or government employees, radical or not, were able to jeopardize the system at times. The way how the peasants acted, for instance, expropriating their own large extensions of land, promoting economic self-management and leading the consolidation of the agrarian reform, alarmed the government in such a way that it was forced, with the approval, or rather the demand of the landowners and the unions, to put an end to the agrarian reform. Therefore, kin to -or influenced by- the different left-wing trends, these social actors replicated, each according to their particularities, the ideological and political tensions of an entire period.

On April 16 ${ }^{\text {th }}, 1972$ the elections for local and departmental posts were held. In a social context marked by the crisis, it was obvious that the political scene would be hardly modified. What was not evident, however, was that the bipartisanship was again unaffected. The traditional parties continued to have their in- fighting, but gave Pastrana an adequate margin to finish his administration term. In the liberal ranks a serious battle between the turbayistas (supporters of Julio C. Turbay) and the lopistas (supporters of Alfonso López) was raised. In the conservative ones, meanwhile, the dispute was between ospinistas (supporters of Mariano Ospina) and the alvaristas (supporters of Álvaro Gómez).The left jumped into the ring with the Revolutionary Independent Labor Movement (Movimiento Obrero Independiente Revolucionario, MOIR). The great defeated was General Rojas Pinilla's movement. Just as it has happened in 1957, fifteen years later -now commanding a new party, and perhaps because the anapista leaders were not able to match their interests to the radicalization of the movement's foundations, or because in several municipalities they were part only a part of a bureaucratic quota- Rojas's group wore out politically. Consequently, without the anapista threat, the National Front confirmed in its last administration that the bipartisan loyalty was still alive, and that, although maintaining a pact of national unity was not necessary anymore, a political bipartisan rearrangement would continue to dominate the rest of the twentieth century, now, of course, within the framework of a new global economic strategy. (Pecaut 2006)

\section{CONCLUSIONS}

The main consequence of the NF was the denial of access of other social sectors to the political race; in fact, instead of "widening the spectrum of the party system", it inclined toward the "absorption of the programs of the opposition movements", and therefore the country "missed the opportunity to build a diversified party system that would have served to boost the political development of the country". His- 
tory would have been different if groups like the Liberal Revolutionary Movement, the National Popular Alliance, the National Democratic Movement, the Christian Democrats, the United Front, the Communist Party and the two guerrilla organizations originated in that time the National Liberation Army (Ejército de Liberación Nacional, ELN) and the Revolutionary Armed Forces of Colombia (Fuerzas Armadas Revolucionarias de Colombia, FARC) - had had access to political participation. Undoubtedly, the 19 April Movement (Movimiento 19 de Abril, M-19) would have lacked a historical justification if the supporters of the National Front had not closed the doors of political participation to their main opposition group, the National Popular Alliance. (Baez, 2006)

\section{REFERENCES}

Acevedo, Á. (2004). El símbolo de un Robin Hood vengado en el occidente de Colombia. Revista Estudios Humanísticos-Historia, (3), 45-66.

Archila, M. (2003). Idas y venidas, vueltas y revueltas: revueltas y protestas sociales en Colombia, 1958-1990. Bogotá: ICANH-CINEP.

Arías, R. (2009). El episcopado colombiano en los años 1960. Revista de Estudios Sociales, (33), 95-158.

Ayala, C. (1999). Credencial Historia.Retrieved on January 31st 2014, from Frente Nacional: acuerdo bipartidista y alternación en el poder: http://www. banrepcultural.org/blaavirtual/revistas/credencial/julio2012/indice

Ayala, C. (2006). El populismo atrapado, la memoria y el miedo: el caso de las elecciones de 1970. Medellín: La Carreta/Universidad Nacional de Colombia.
Báez, A. (2006). La Alianza Nacional Popular (Anapo) en Santander, 19621976. Bucaramanga: Universidad Industrial de Santander.

Berry, A. (2012). La tragediade la reforma agraria del Frente Nacional. In C. Caballero, M. Pachón Buitrago, \& E. Posada Carbó, Cincuenta años de regreso a la democracia: nuevas miradas a la relevancia histórica del Frente Nacional (pp. 295-323). Bogotá: Uniandes/Escuela de gobierno Alberto Lleras Camargo.

Calle, H. (2012). El plebiscito de 1957, la legitimidad fundacional del Frente Nacional. In C. C. otros, Cincuenta años de regreso a la democracia: nuevas miradas a la relevancia histórica del Frente Nacional (pp. 119-146). Bogotá: Uniandes/Escuela de Gobierno Alberto Lleras Camargo.

Colombia, Congreso de la República de (1961). Ley 135 Sobre reforma social agraria. Bogotá DC: Diario Oficial 30691

Hartlyn, J. (1993). La política del Régimen de coalición: La experiencia del Frente Nacional en Colombia. Bogotá: Centro de Estudios Internacionales de la Universidad de los Andes (CEI)/ Uniandes/Tercer Mundo Editores.

Henderson, J. (1984). Cuando Colombia se desangró: un estudio de la violencia en metrópoli y provincia. Bogotá: El Áncora.

Henderson, J. (2006). La modernización en Colombia: los años de Laureano Gómez, 1889-1965. Medellín: Universidad de Antioquia.

Junguito, R. \& Rincón, F. (2007). La política fiscal en el siglo XX en Colombia. In J. Robinson, \& M. Urrutia, Economía colombiana del siglo $X X$ : un análisis cuantitativo (pp. 239-308). Bogotá: Fondo de Cultura Económica. 
Kalmanovitz, S. (1995). Economía y nación, una breve historia de Colombia. Bogotá: Tercer Mundo.

Leal, F. (1984). La participación política de la juventud universitaria como expresión de clase. In M. Cárdenas, \& A. Díaz Uribe, Juventud y política en Colombia (pp. 155-203). Bogotá: FESCOL/Instituto SER.

Machado, A. (1998). La cuestión agraria en Colombia a fines del milenio. Bogotá: El Áncora.

Machado, A. Centro de Estudios Estadounidenses Colombia. Retrieved on: http://www.ceecolombia.org/ckfinder/userfiles/files/La\%20alianza\%20para $\% 20$ el\%20progreso $\% 20$ y\%20la\%20reforma\%20agraria\%20 (Absal\%C3\%B3n\%20Machado).pdf

Mayorga, F. (2013). Historia de la organización electoral en Colombia (18882012): vicisitudes de la consolidación democrática en un país complejo. Bogotá: Universidad del Rosario/Facultad de Jurisprudencia.

Musgrave, R. (1969). Informe Musgrave: bases para una Reforma Tributaria en Colombia. Bogotá: Biblioteca Banco Popular.

Palacios, M. (1998). Entre la legitimidad y la violencia, Colombia, 1875-1994. Bogotá: Norma.
Palacios, M. (2012). Violencia pública en Colombia, 1958-2010. Bogotá: Fondo de Cultura Económica.

Pecaut, D. (2006). Crónica de cuatro décadas de política colombiana. Bogotá: Norma.

Plazas, M. (2011). El Frente Nacional. Bogotá: Temis.

Ramírez, M. \& Tellez, J. (2007). La educación primaria y secundaria en Colombia en el siglo XX. In J. Robinson, \& M. Urrutia, Economía colombiana del siglo XX: un análisis cuantitativo (pp. 459-513). Bogotá: Fondo de Cultura Económica.

Restrepo, J. D. (1995). La revolución de las sotanas: Golconda 25 años después. Bogotá: Planeta.

Tirado, Á. (1981). Colombia: siglo y medio de bipartidismo. In M. Arrubla, Colombia hoy (pp. 100-189). Bogotá: Siglo XXI.

Urrutia, M. (2012). Política económica y social en el régimen de coalición. In Caballero, \& C. y. Caballero, Cincuenta años de regreso a la democracia: nuevas miradas a la relevancia histórica del Frente Nacional (pp. 241-258). Bogotá: Uniandes/Escuela de gobierno Alberto Lleras Camargo.

Vargas, A. (1996). Política y armas al inicio del Frente Nacional. Bogotá: Universidad Nacional de Colombia. 